\title{
Multiple-pattern learning by rats on an eight-arm radial maze
}

\author{
C. NADINE WATHEN and WILLIAM A. ROBERTS \\ University of Western Ontario, London, Ontario, Canada
}

\begin{abstract}
Rats were trained over a number of sessions on an eight-arm radial maze with eight trials on each session. Each of four arms on the maze contained a different pattern formed by sequences of reward (two pellets) or nonreward (no pellets) over successive trials within sessions. The patterns were single alternation, double alternation, and two patterns in which four rewards or four nonrewards were preceded and followed by two nonrewards or two rewards, respectively. The other four arms on the maze served as control arms and always contained one pellet. It was found that rats tracked all of these patterns when they were required to climb over barriers to enter and leave arms. However, rats showed no ability to extrapolate patterns beyond the training trials. These findings, and a further analysis of arm-choice stereotypy, led to the conclusion that rats tracked by using a trial-number strategy.
\end{abstract}

Phelps and Roberts (1991) reported three experiments in which quantity of reward was varied across trials on each arm of a four-arm radial maze within daily sessions. It was found that rats could track two patterns simultaneously over successive trials. In two experiments, these patterns consisted of either (1) long strings of reward or nonreward or (2) single- and double-alternation patterns of reward and nonreward. In another experiment, monotonically increasing and decreasing patterns of reward magnitude were used. Pattern tracking was measured by the order in which arms of the maze were entered within successive trials. Accurate tracking of these predictable patterns was indicated by low ranks of arm entry on trials when an arm contained reward and high ranks of arm entry on trials when an arm contained nonreward. Patterns that changed in a less predictable, quasi-random manner were not well tracked.

Tracking on the radial maze was studied further in three experiments reported here, in which procedures different from those employed by Phelps and Roberts were used. The four-arm radial maze used by Phelps and Roberts puts a severe limitation on the number of patterns that can be studied. Problems arise if four predictable patterns are used, one assigned to each arm of the maze. All four patterns may program a reward on the same trial. Because the arms of the maze can only be entered in sequential order, it is necessary that one rewarded arm will be entered first and be assigned the lowest possible rank of 1 , whereas another rewarded arm will be

Support for this research was provided by a research grant from the Natural Sciences and Engineering Research Council of Canada to W. A. Roberts. Experiment 1 was reported by C. N. Wathen as part of an M.A. thesis submitted to the University of Western Ontario. Correspondence should be addressed to W. A. Roberts, Department of Psychology, University of Western Ontario, London, ON, Canada N6A 5C2. entered last and be assigned the highest rank of 4 . A similar problem arises if all four patterns should program a nonreward on the same trial.

To alleviate this problem, the present experiments used an eight-arm radial maze, with four of the arms designated as control arms. The control arms always contained one food pellet on every trial of a session. The remaining four arms of the maze were assigned patterns that changed predictably between reward (two pellets) and nonreward (no pellets) over successive trials. This procedure allowed ranks of arm entry to vary from 1 to 8 and meant that multiple arms containing reward or nonreward on the same trial could all achieve relatively low or high ranks.

One major question then addressed by these experiments was whether rats could simultaneously track more than two patterns on the radial maze. The opportunity to track as many as four patterns was made available by the use of the eight-arm maze. The difficulty of pattern tracking in these experiments was increased over the Phelps and Roberts (1991) situation not only by increasing the number of arms containing patterns but also by using patterns that varied in the rules that dictated them. In the Phelps and Roberts study, similar rules controlled the generation of both tracked patterns within an experiment. In the present experiments, two patterns were used that always required a repeat rule, single and double alternation. The other two patterns that were simultaneously presented required a switch between reward and nonreward at Trials 3 and 7.

The theoretical approach favored by Phelps and Roberts (1991) to explain multiple tracking on the radial maze was to suggest that rats had learned two patterns, which could be assigned to and tracked on separate arms of the maze. It was argued that either Capaldi's theory of sequential associations between memory chunks (Capaldi, 1992; Capaldi \& Verry, 1981) or Fountain and Hulse's rule learning theory (Fountain, 1990; Fountain, Henne, \& 
Hulse, 1984) could potentially account for pattern learning on the radial maze. This general approach can be called the arm-pattern hypothesis.

An alternative account is the trial-number hypothesis. According to this hypothesis, rats learn to expect reward in certain arms on each successive trial. The ordinal position or number of a trial serves to control the rat's armentry preferences. The appearance of pattern tracking then arises from the rat's learning simply to enter certain rewarded arms first on each trial. Recent suggestions that rats may be capable of some degree of numerical competence (Davis \& Perusse, 1988) or may be able to count (Capaldi \& Miller, 1988; Gallistel, 1990) encourage the possibility that trial number may be an important source of pattern acquisition information.

The present experiments address these hypotheses in several ways. First, the use of four different patterns, with different types of rules used to generate pairs of patterns, should put a considerable cognitive burden upon the use of an arm-pattern strategy. Therefore, we might expect that if rats track by learning the pattern on each arm, these patterns would not be learned or would be only partially learned. The trial-number hypothesis, on the other hand, predicts tracking of all patterns, because the rat would learn the rewarded arms of each pattern to be entered first on each trial.

It will be shown that the results of Experiment 1 yielded evidence of only partial tracking, which might suggest that the task had become too difficult for rats to learn. In Experiment 2 , the possibility that increased effort to enter arms of the maze might improve tracking was examined by presenting rats with the same patterns used in Experiment 1 but with the additional requirement that rats climb a barrier to enter the arms. Brown (1990; Brown \& Huggins, 1993; Brown \& Lesniak-Karpiak, 1993) has found that rats more accurately avoid errors on radial maze arms when the effort required to enter these arms has been increased. He suggests that improved accuracy under effortful conditions can best be understood as a shift in choice criterion.

As a second issue bearing on these two hypotheses, armentry data were examined for agreement in the order in which specific arms were entered within trials over successive sessions. Although the arm-pattern hypothesis is silent on the question of arm order, the trial-number hypothesis holds that rats learn strong preferences for certain alleys on each trial; sequences of arm entries within trials might become stereotyped over sessions. The summation of arm-direction vectors was used to measure stereotypy of response patterns across sessions.

Finally, the third experiment looked for the extrapolation of patterns that had been learned over previous sessions involving a fixed number of trials. The arm-pattern hypothesis suggests that repeating patterns should be extrapolated. On the other hand, the trial-number hypothesis does not predict extrapolation because arm preferences have been learned only up to the number of trials used in training.

\section{EXPERIMENT 1}

Four patterns were assigned to four randomly chosen arms of an eight-arm radial maze, with these assignments remaining constant throughout training. The four remaining arms were designated control arms and always contained one pellet on each trial. The patterns used are shown in Table 1, and each consisted of eight elements or trials. Two of these were repeating patterns, single alternation (SA) and double alternation (DA), and the other two consisted of either a block of two nonrewards followed by four rewards and a final block of two nonrewards (2N4R2N) or a block of two rewards followed by four nonrewards and a final block of two rewards (2R4N2R). Each maze arm, whether a patterned or control arm, yielded a total of eight pellets per session. Therefore, no arm preferences should have developed as a consequence of arm differences in total food obtained. The question of major importance in this experiment was whether or not rats could learn to track all four of the patterns used.

\section{Method}

Subjects. Six male Long-Evans hooded rats (Rattus norvegicus) served as subjects. They were approximately 120 days old at the start of the experiment. The animals were housed separately in wire cages and were exposed to a 16:8-h light:dark schedule, with light onset at 6 a.m. and offset at 10 p.m. All animals were kept at $85 \%$ of their free-feeding weight.

Apparatus. An unwalled eight-arm radial maze, made of plywood and painted flat black, was used for all pretraining and training sessions. Each of the eight arms was $61 \mathrm{~cm}$ in length and $8 \mathrm{~cm}$ wide and radiated from an octagonal center platform $36 \mathrm{~cm}$ in diameter. Pieces of wooden doweling supported the maze at a height of $60 \mathrm{~cm}$ above the floor. Round plastic food wells $(2.5 \mathrm{~cm}$ in diameter, $0.5 \mathrm{~cm}$ deep) were located $1 \mathrm{~cm}$ from the end of each arm. These wells were covered by the experimenter with square metal plates that measured $4 \times 4 \mathrm{~cm}$ and weighed $75 \mathrm{~g}$. These covers concealed the contents of the food wells and reduced any olfactory cues that the animals might use to choose rewarded arms. The rewards used were $45-\mathrm{mg}$ Noyes pellets.

The maze was located in an experimental room measuring $3.2 \times$ $2.8 \mathrm{~m}$. Light was provided by two overhead fluorescent lights. The room contained numerous spatial cues, including a table, pictures on the walls, and several large pieces of equipment. The experimenter sat in a corner approximately $1 \mathrm{~m}$ from the nearest maze arm. Responses were recorded by hand on data sheets indicating the order of arm entry within each trial for each animal. A masking noise was played by a white-noise generator during all training sessions.

Procedure. The animals initially received 14 days of habituation to the maze environment and pretraining on the maze. The subjects were trained initially to visit the food wells located at the end

Table 1

Patterns of Reward (R) and Nonreward (N) Used in Experiments 1, 2, and 3

\begin{tabular}{lllllllll}
\hline & \multicolumn{10}{c}{ Trial } \\
\cline { 2 - 8 } Pattern & 1 & 2 & 3 & 4 & 5 & 6 & 7 & 8 \\
\hline SA & N & R & N & R & N & R & N & R \\
DA & R & R & N & N & R & R & N & N \\
2N4R2N & N & N & R & R & R & R & N & N \\
2R4N2R & R & R & N & N & N & N & R & R \\
\hline
\end{tabular}


of each arm to obtain one food pellet and then on subsequent sessions to push aside the metal cover on the food well to obtain the pellet.

Training sessions consisted of eight trials per day; the animals were tested 6 days per week. For each subject, a different random assignment of the arms of the maze was made, with four arms assigned to the four patterns shown in Table 1 and the other four arms designated control arms. Each pattern then was repeated on the same arm for a given subject throughout 40 sessions of training. On each trial, the four control arms each contained one 45-mg Noyes pellet, and the four pattern arms each contained either reward ( $R$; two pellets) or nonreward (N; no pellets) according to its assigned pattern.

Each trial began with placement of the subject on the center of the maze. The experimenter recorded arm choices in order until all eight arms had been visited. The rat then was removed from the maze and put into an opaque holding chamber with water freely available. The experimenter then rebaited the arms of the maze over an intertrial interval of approximately $30 \mathrm{sec}$ and placed the rat on the center for the next trial. Once the subjects had learned to perform efficiently, overall session length was approximately $15 \mathrm{~min}$.

All analyses reported in these experiments were considered significant if $p<.05$.

\section{Results and Discussion}

The data were analyzed in 10 blocks of four sessions each. Within each block, a measure of pattern-tracking accuracy was calculated for each subject on each pattern. This measure was obtained by subtracting the mean rank of arm entry on rewarded trials from the mean rank of arm entry on nonrewarded trials. A high positive difference score indicated early choice of rewarded arms and late choice of nonrewarded arms within trials, whereas a score of zero indicated indifference between rewarded and nonrewarded arms. Mean difference scores are shown as a function of blocks of trials for each pattern in Figure 1 . The scores for each pattern stay near zero through Blocks 3 and 4 and then begin to rise over subsequent blocks. Although all four patterns show a rise in difference scores, the SA pattern rises considerably more than the other three. A subject $\times$ block $\times$ pattern analysis of variance (ANOVA) yielded significant effects of block $[F(9,200)=23.18]$, pattern $[F(3,200)=14.22]$, and the block $\times$ pattern interaction $[F(27,200)=2.97]$. A Newman-Keuls post hoc analysis on the data from Block 10 showed that the SA pattern was significantly higher than each of the other three patterns, which did not differ among themselves. In general, the curves suggest that all four patterns were acquired to some extent, but the SA pattern was better learned than the other three.

In Figure 2, tracking performance is depicted for each pattern across the eight trials of each session in Block 10 . Each panel contains the same control curve for comparison purposes. The control curve is based on the mean rank of entrance into all four of the control arms. An ANOVA showed no significant effect of trial on mean rank of entrance to control arms $(F<1.0)$. The curve for the SA pattern, shown in the upper left panel, indicates that the animals regularly tracked this alternating pattern of reward and nonreward. The mean rank on nonrewarded trials was significantly higher than the mean rank on rewarded trials $[t(5)=7.96]$, and all of the mean differ-

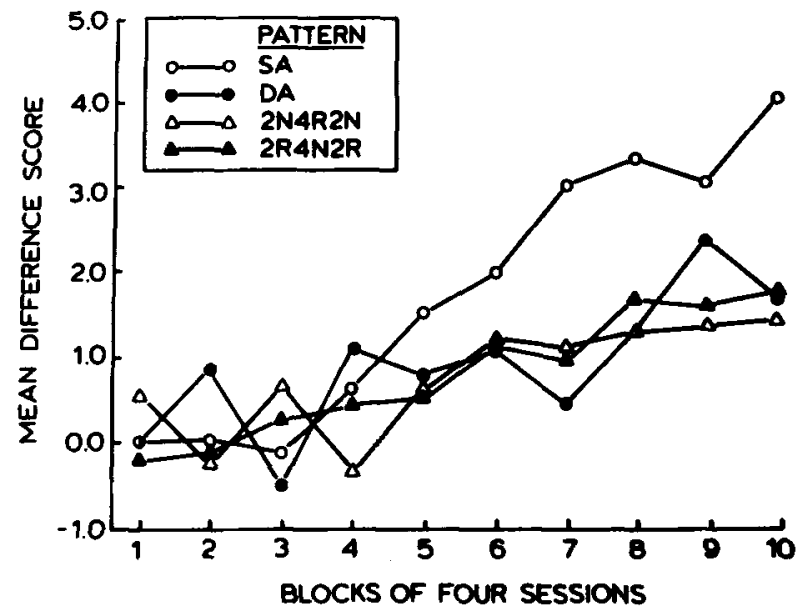

Figure 1. Acquisition of pattern tracking shown for four patterns over 10 blocks of four sessions each in Experiment 1. Mean difference score refers to the difference between entry ranks on nonrewarded and rewarded trials.

ences between rewarded and nonrewarded adjacent trials were significant $[t(5) \geq 2.31]$. In the DA curve shown in the upper right panel, tracking is far less obvious. The strongest indication of pattern sensitivity in this curve is the drop in mean rank on Trial 5, the first $R$ trial after the initial RRNN sequence. The overall mean rank on $R$ trials was significantly lower than the overall mean rank on $N$ trials in Pattern DA $[t(5)=3.40]$, but the only significant difference between trials occurred between Trials 4 and $5[t(5)=4.68]$.

Data from Pattern 2N4R2N, shown in the lower left panel of Figure 2, indicate pattern learning only on Trial 3, where there is a sharp drop in mean rank at the first $R$ after the initial NN sequence. As a consequence primarily of the effect at Trial 3, the difference between overall mean rank on $\mathbf{N}$ and $\mathbf{R}$ trials was significant $[t(5)=9.48]$. Also, the drop in mean rank between Trials 2 and 3 was significant $[t(5)=4.77]$. Finally, the lower right panel presents data from the $2 R 4 N 2 R$ pattern, which indicate a higher overall mean rank for $\mathrm{N}$ than for $\mathrm{R}$ trials $[t(5)=3.90]$. The most marked sensitivity to the pattern was found in the significant drop at Trial 7 , the first $\mathbf{R}$ trial after the RRNNNN sequence $[t(5)=2.98]$.

A comparison of performance on pattern arms and control arms indicated that mean rank on rewarded arms was significantly lower than that on control arms for Patterns SA $[t(5)=23.37]$, DA $[t(5)=3.26]$, and $2 \mathrm{~N} 4 \mathrm{R} 2 \mathrm{~N}$ $[t(5)=4.46]$, but not for Pattern 2R4N2R $[t(5)=1.89]$. There were no significant differences between entry ranks to nonrewarded and control arms for any pattern.

In general, the data shown in Figure 2 suggest that the rats fully acquired the SA pattern but only partially acquired the other three patterns. A rule that summarizes the findings is that the rats always showed a clear drop in mean entry rank on the first rewarded trial to follow 

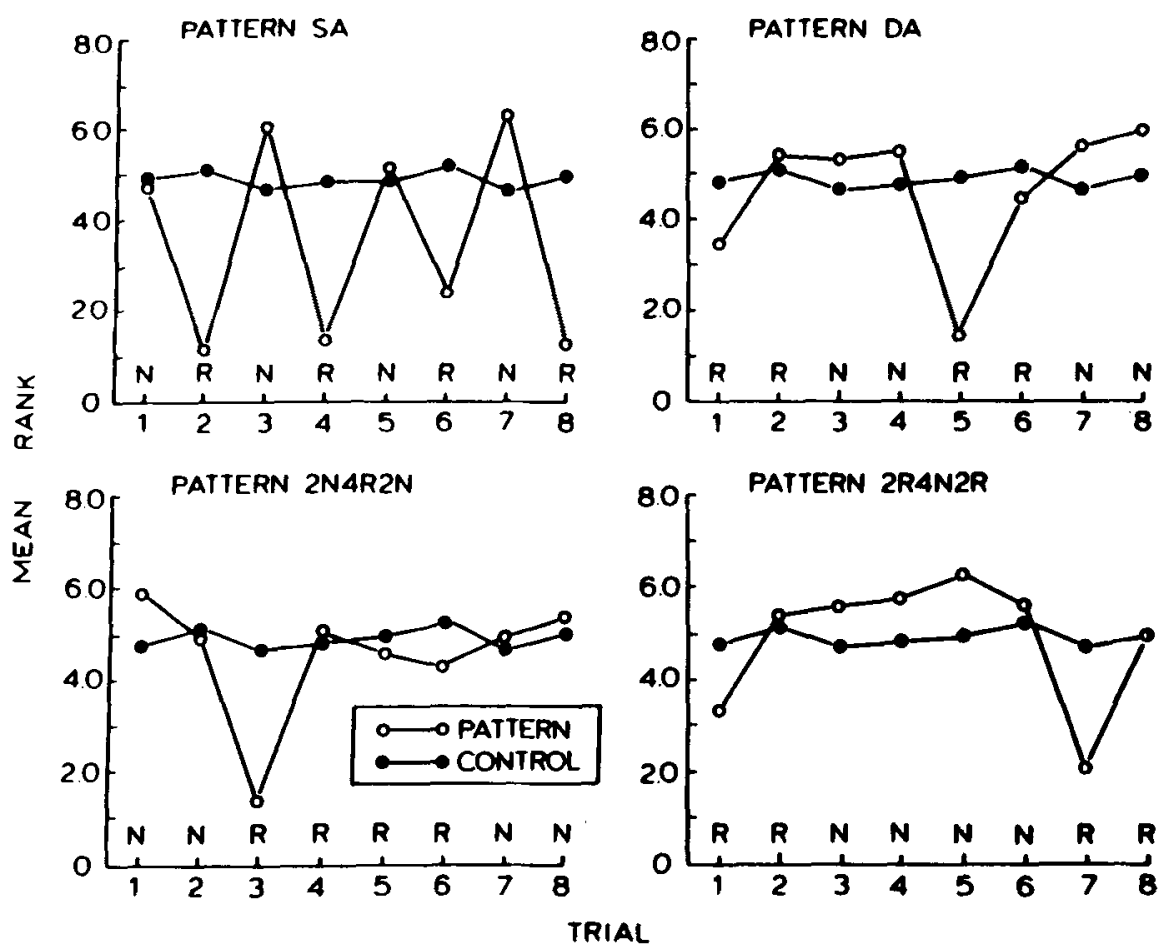

Figure 2. Mean rank of entry into rewarded $(R)$ and nonrewarded $(N)$ arms for each pattern. The data shown are from Block 10 of Experiment 1.

a nonrewarded trial. This rule yields strong tracking throughout the SA pattern but clear evidence of tracking only at Trial 5 of the DA pattern, Trial 3 of the 2 N4R2N pattern, and Trial 7 of the 2R4N2R pattern.

These data were analyzed further to determine what response patterns the animals developed to partially track the four patterns. Typically, it has been found that rats on a radial maze display response patterns of entering adjacent arms or every second arm in a clockwise or counterclockwise direction, with random choice of arms only occasionally seen (Dale, 1986; Foreman, 1985; Higashida \& Ogawa, 1987). In the present experiment, 5 of the subjects showed an early tendency to enter every second arm, and the remaining subject tended to enter adjacent arms. As sessions proceeded, these patterns became modified. Relative preferences for adjacent or alternate arms were replaced by absolute preferences for rewarded arms, and response patterns were repeated over sessions.

A circular statistic was used to measure the development of response stereotypy across sessions. The radial maze represents a circular surface with eight possible response directions. Each response can be thought of as a vector with a length of 1.0 and a definable direction. For this analysis, overall response vectors were determined by adding response vectors over sessions at each of the first eight arm choices within trials and calculating the mean resultant vector. A computer program was used that added the $x$ and $y$ coordinates of arms chosen over a fixed block of sessions and determined the length of the vector indicated by the mean coordinates. Mean resultant vectors could vary between 0 and 1.0 in length, with higher vectors indicating greater response stereotypy. Thus, if the same arm was chosen over all the sessions being analyzed, the mean resultant vector would be 1.0. If different arms were chosen on each session, the mean resultant vector would be zero.

Vector length was determined for three separate phases of acquisition, early (Blocks 1-3), middle (Blocks 4-7), and late (Blocks 8-10). Mean vector lengths are shown as a function of arm choice for each block of sessions in Figure 3. The rise in the level of the curves over early, middle, and late sessions clearly indicates that response patterns became more stereotyped as sessions progressed. The curves further suggest that the highest levels of stereotypy were found on initial choices within trials and declined on later choices. Statistical analyses of these curves indicated significant main effects of choice number, $[F(7,120)=28.75]$ and acquisition phase $[F(2,120)=$ $52.99]$, as well as a significant interaction of choice number $\times$ acquisition phase $[F(14,120)=2.62]$. NewmanKeuls post hoc tests indicated that mean vector lengths differed significantly among all three acquisition phases at arm choices $1,2,3,7$, and 8 . At arm choices 4,5 , and 6 , late acquisition vectors were significantly longer than both early and middle acquisition phase vectors, which did not differ significantly from one another.

In order to determine whether mean vector lengths exceeded those expected by chance, 96 sequences of the 


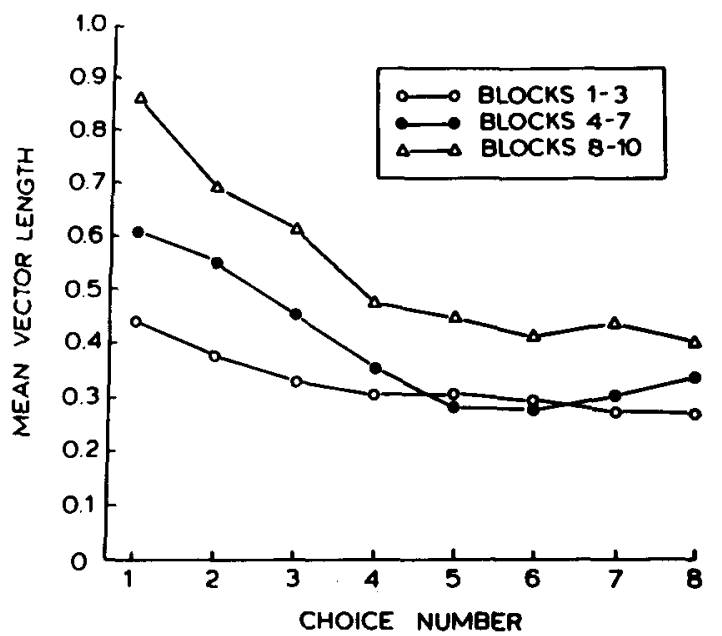

Figure 3. Mean vector length plotted as a function of Choices 1-8 within trials. Separate curves are shown for Blocks 1-3 (early), Blocks 4-7 (middle), and Blocks 8-10 (late) of Experiment 1.

numbers 1-8 were randomly generated by computer and put into the vector addition program in blocks of eight sequences. The resultant vector lengths were averaged, and they yielded a mean chance value of .26 . Observed mean vectors for Choices $1-4$ and 5-8 were statistically compared with the chance value of .26 at each acquisition phase. The mean vectors for Choices 1-4 significantly exceeded chance at early, middle, and late acquisition phases $[t(5) \geq 3.86]$. For Choices 5-8, mean vector length was significantly higher than chance at the middle and late acquisition phases $[t(5) \geq 2.86]$; however, vector length in the early acquisition phase did not differ significantly from chance $[t(5)=1.66]$.

The findings from Experiment 1 showed that the rats were able to track four different patterns on an eight-arm maze to a limited extent. Patterning behavior could be described by the general rule that the rats were highly sensitive to any rewarded trial that followed a nonrewarded trial. These occasions gave rise to a sharp drop in armentry rank on the final acquisition sessions. However, rewarded trials that followed immediately preceding rewarded trials were not tracked with a low arm-entry rank, and entry ranks on nonrewarded trials in general were not higher than the mean entry ranks on control arms. These findings then are somewhat at variance with those of Phelps and Roberts (1991), who found accurate tracking both of SA and DA patterns and of embedded strings of five rewarded or nonrewarded trials.

The other important aspect of these findings is the discovery that the development of pattern tracking was accompanied by the development of sequences of arm choices that were stereotyped or highly repetitive over successive sessions. It is possible that what pattern tracking was seen in this experiment was dependent upon the development of relatively fixed and inflexible sequences of arm choices on each trial.

\section{EXPERIMENT 2}

The failure of subjects to show consistent tracking of all four patterns in Experiment 1 may reflect limits on rats' ability to learn multiple patterns. If rats tend to learn a separate pattern on each arm, as the arm-pattern hypothesis suggests, learning to track four patterns accurately may be beyond these animals' ability. The observation that rats seemed to discriminate only rewarded trials that followed nonrewarded trials may represent the best solution that they were able to find.

Before concluding that the limit of the rat's ability to track multiple patterns has been reached, however, another possibility should be considered. In the experiments carried out by Phelps and Roberts (1991), rats were required to climb over a barrier both in order to enter an arm and to return to the center of the maze. In radial maze foraging experiments, the use of barriers increases travel time and strongly affects foraging behavior, causing rats to search patches exhaustively and to carry food less often to the center of the maze (Ash \& Roberts, 1992; Phelps \& Roberts, 1989; Roberts \& Ilersich, 1989). It may be that this requirement of additional time and effort to enter and exit arms reduces precipitous arm-entry decisions by raising the choice criterion (Brown \& Lesniak-Karpiak, 1993) and thereby sharpens pattern tracking. In order to find out if extra time and effort to enter arms of the maze improves tracking performance, Experiment 1 was replicated in Experiment 2 with barriers placed at arm entrances.

\section{Method}

Subjects. Six naive male Long-Evans hooded rats served as subjects. They were approximately 120 days old at the start of the experiment. The animals were housed under the same conditions as those in Experiment 1.

Apparatus. A different maze and testing room from those used in Experiment 1 were used in Experiment 2. An eight-arm plywood radial maze, black in color, was used to pretrain and train subjects. This unwalled maze stood $61 \mathrm{~cm}$ above the floor and had arms $74 \mathrm{~cm}$ long $\times 9 \mathrm{~cm}$ wide $\times 1.5 \mathrm{~cm}$ thick, which radiated from a 36-cm-diameter center. Food wells $(2.5 \mathrm{~cm}$ in diameter, $1 \mathrm{~cm}$ deep) were located $3.5 \mathrm{~cm}$ from the end of the arms. These wells were covered with the same metal plates used in Experiment 1.

Wooden barriers $(12.5 \mathrm{~cm}$ wide $\times 15 \mathrm{~cm}$ high $\times 5 \mathrm{~cm}$ deep) were added to the maze. A barrier was placed at the entrance to each of the eight arms and was attached to the arm by a bolt that went through a hole in the arm, allowing the barrier to be fixed to the arm with a wing nut. A nonreported experiment carried out with another group of animals on this apparatus showed that rats tended to cross between arms either near the arm entrance or by walking around the tops of the barriers. This behavior was prevented in this experiment first by attaching a $20 \times 14 \times 0.25 \mathrm{~cm}$ piece of plywood lengthwise to one side of each arm, beginning $5 \mathrm{~cm}$ from the arm entry to allow room to attach the wooden barriers. Second, black posterboard baffles were attached to the side of each barrier. These baffles extended $10 \mathrm{~cm}$ above the top of the barrier and prevented rats from walking across the tops of the barriers.

The maze was located in a $3.1 \times 3.1 \mathrm{~m}$ room with two overhead fluorescent lighting units and several extramaze cues to distinguish the walls of the room from one another. The experimenter sat in a corner and recorded each trial's data. The subjects were placed 


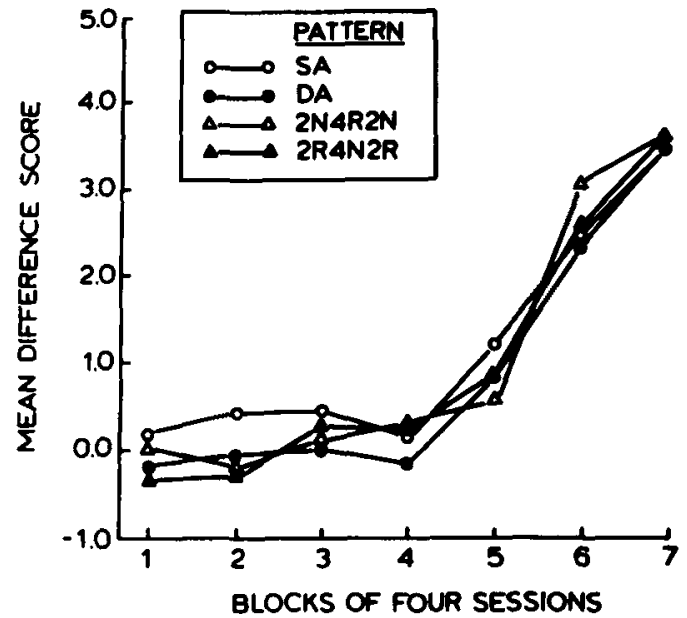

Figure 4. Acquisition of pattern tracking shown for four patterns over seven blocks of four sessions each in Experiment 2. Mean difference score refers to the difference between entry ranks on nonrewarded and rewarded trials.

in the same chamber as the one used in Experiment 1 during approximately $30-\mathrm{sec}$ intertrial intervals while the experimenter rebaited the food cups.

Procedure. During 14 days of habituation and pretraining, the rats were trained to run out on the arms and push the food-cup cover aside to obtain one 45-mg Noyes pellet. In addition, each subject was trained to climb over the barriers in order to enter and leave each arm. The barriers were introduced gradually by requiring the animals first to climb over barriers placed face down, then placed on their sides, and finally placed at full height.
The rats were trained over a period of 28 daily sessions. Each rat had a different random assignment of the SA, DA, 2N4R2N, and $2 R 4 N 2 R$ patterns to four arms of the maze, with the other four arms designated control arms. Each session consisted of eight successive trials, with the amounts of reward placed in the food cups and the testing procedures used corresponding to those used in Experiment 1.

\section{Results and Discussion}

Difference scores, consisting of mean rank of entry on nonrewarded trials minus mean rank of entry on rewarded trials, were calculated for each pattern over seven blocks of four sessions each. Acquisition curves depicting these difference scores over blocks of sessions are shown in Figure 4. These curves suggest that little improvement in tracking occurred over the first four blocks of sessions, but steady improvement up to a substantial level of tracking appeared over Blocks 5-7. Furthermore, the rates of improvement and the final levels of tracking appear to be equivalent for all four patterns. This impression was substantiated by an ANOVA that yielded a significant effect of blocks of sessions $[F(6,140)=45.30]$ but no significant effects of type of pattern or the block $\times$ type of pattern interaction $(F \mathrm{~s}<1.00)$.

Tracking of individual patterns is shown in Figure 5, with mean rank of entry into all four control arms on each trial plotted for comparison with the mean ranks for each pattern. Although the control curve appears to change little over trials, its variation in mean rank over trials was significant $[F(7,40)=6.17]$. All four patterns show strong consistent tracking on all trials. Mean rank of entry on $R$ trials was significantly lower than mean rank of entry
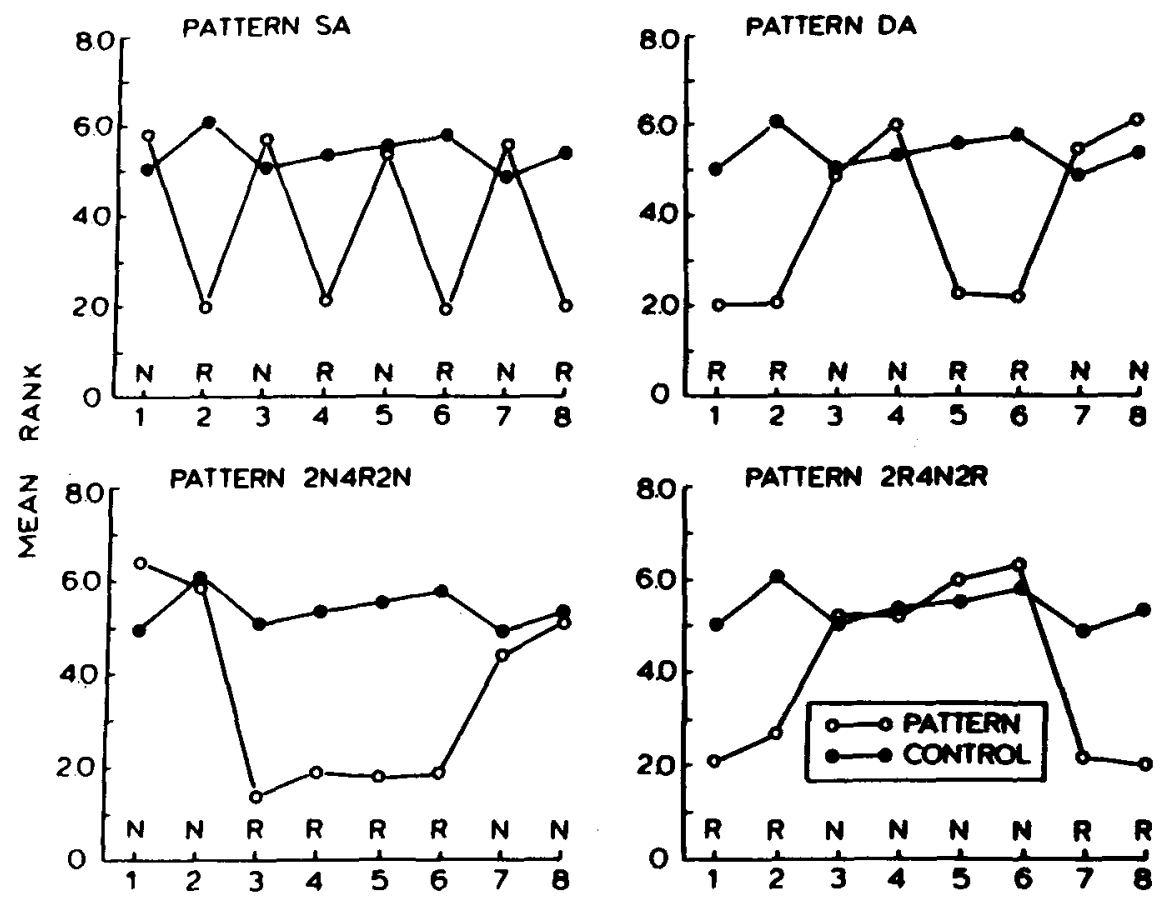

TRIAL

Figure 5. Mean rank of entry into rewarded $(R)$ and nonrewarded $(N)$ arms for each pattern. The data shown are from Block 7 of Experiment 2. 
on $\mathrm{N}$ trials for all four patterns $[t(5) \geq 8.56]$. The differences in mean rank between trials that involved transitions from $N \rightarrow R$ and $R \rightarrow N$ were significant on all patterns $[t(5) \geq 2.84]$. Finally, mean ranks on $\mathrm{R}$ trials were significantly lower than the mean rank of control trials for all four patterns $[t(5) \geq 11.25]$, but mean ranks on $\mathrm{N}$ trials never differed significantly from that on control trials $[t \mathrm{~s} \leq 1.32]$.

The data clearly show that the rats strongly tracked rewarded trials (two pellets) by entering these alleys early in a trial. By contrast, nonrewarded arms (no pellets) were treated the same as control arms (one pellet), with both entered at mean ranks between 5.0 and 6.0. As a further analysis of tracking behavior, vector addition scores were calculated for each subject on the first eight trials of a session over early sessions (Blocks 1 and 2), middle sessions (Blocks 3-5), and late sessions (Blocks 6 and 7). A plot of mean resultant vector length against Choices 1-8, shown in Figure 6, indicates that (1) stereotypy was highest on the early choices and dropped across choices and (2) stereotypy increased across sessions, as the patterns were acquired. An ANOVA carried out on vector scores yielded significant main effects of choice number $[F(7,120)=$ 28.03], acquisition phase $[F(2,120)=14.10]$, and choice number $\times$ acquisition phase $[F(14,120)=2.02]$. The mean vector length over Choices 1-4 and Choices 5-8 was compared at each acquisition phase with the resultant vector length of . 26 expected by chance. Stereotypic behavior was found to be significantly above chance on both blocks of choices at all three stages of acquisition $[t s(5) \geq 4.12]$, with one exception: Mean vector length for Choices 1-4 in the middle acquisition phase did not significantly exceed the chance value $(t<1.00)$.

In general, the vector analysis provided findings similar to those of Experiment 1. The animals became pro-

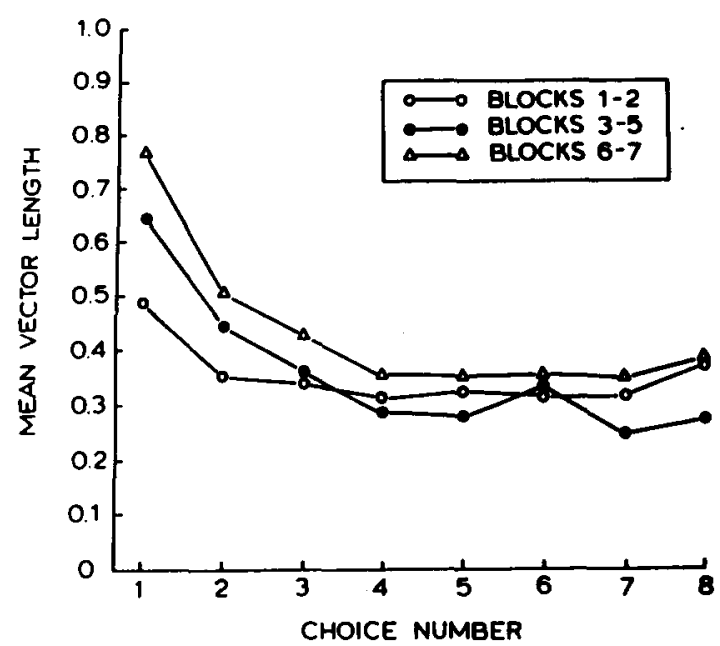

Figure 6. Mean vector length plotted as a function of Choices 1-8 within trials. Separate curves are shown for Blocks 1 and 2 (early), Blocks 3-5 (middle), and Blocks 6 and 7 (late) of Experiment 2. gressively more stereotyped in their sequence of alley choices on each trial over sessions during which pattern acquisition was occurring. Stereotypy was more marked on the early choices of trials than on the later choices.

\section{EXPERIMENT 3}

Experiment 3 was designed to test whether rats could extrapolate patterns beyond the 8 trials given in each session throughout acquisition. Previous experiments have shown that rats can track SA patterns in a runway for 12 or more trials (Bloom \& Capaldi, 1961). Four rats that completed Experiment 2 were given four sessions consisting of 12 trials. On the first 8 trials, the subjects were rewarded on pattern and control arms, as they were in acquisition. The question of what rewards should be available on arms during the 4 extrapolation trials was problematic. In an experiment carried out by Fountain and Hulse (1981), extrapolation of a monotonically decreasing pattern of reward magnitude was found on a single extrapolation trial involving nonreward. In that experiment, the use of an extinction trial conveniently continued the training pattern. However, the use of 4 extinction trials with no arms baited in the present experiment might simply lead to extinction of performance. Therefore, we chose to continue to reward the animals according to the acquisition patterns. On Trials 9-12, the patterns of reward were NRNR on the SA arm, RRNN on the DA arm, RRRR on the 2N4R2N arm, and NNNN on the 2R4N2R arm.

It was reasoned that the arm-pattern and trial-number hypotheses predict different outcomes for the extrapolation test. If animals used arm patterns based on sequential associations or rules, it would seem that they should be able to extrapolate patterns beyond the final trial of the acquisition sessions. Particularly with respect to the SA and DA patterns that repeated within sessions, the use of sequential associations or rules should allow rats to continue the pattern. The trial-number hypothesis, on the other hand, clearly predicts the absence of pattern extrapolation. Without having learned any response sequences for Trials 9-12, animals should show little systematic preference between arms across trials.

\section{Method}

Subjects and Apparatus. Of the 6 rats that were tested in Experiment 2, 4 rats began and completed that experiment several weeks before the remaining 2 rats. These 4 rats were given four sessions of extrapolation testing in Experiment 3. Time limitations prohibited testing of the remaining 2 rats. The animals tested in Experiment 3 continued to be housed and fed in the same way as they had been during Experiment 2. The apparatus and testing room were identical to those used in Experiment 2.

Procedure. On each of four sessions, each rat was returned to the maze for 4 trials beyond the 8 normally given during the 28 sessions of acquisition. The pattern arms again contained rewards and nonrewards according to a continuation of the 8-trial training pattern, and the control arms again contained one pellet on each trial. All other procedural and data collecting aspects of the experiment were the same as those used in Experiment 2. 

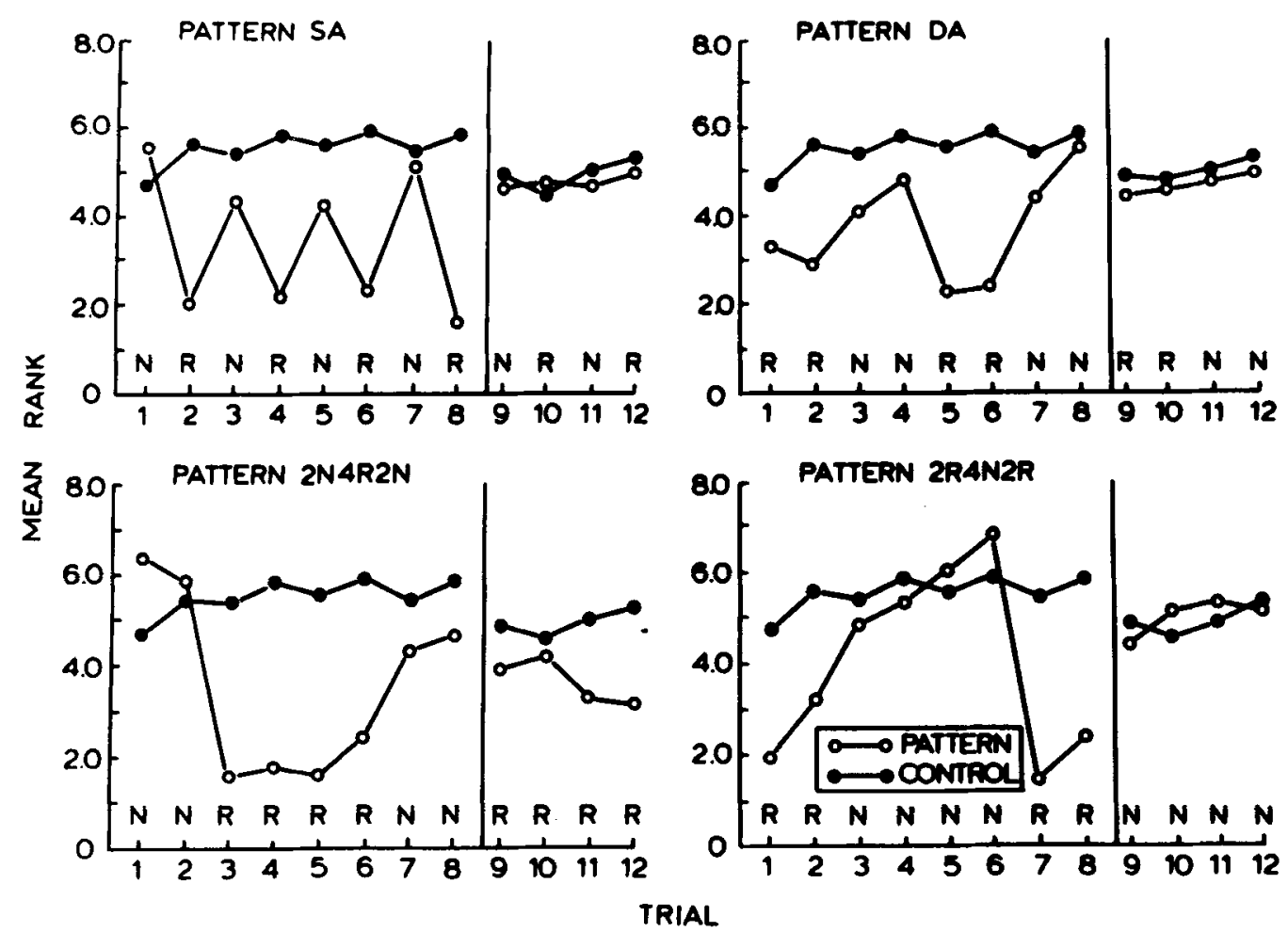

Figure 7. Mean rank of entry into rewarded $(R)$ and nonrewarded (N) arms for each pattern. The data are from Experiment 3 and show performance on Extrapolation Trials 9-12.

\section{Results and Discussion}

Figure 7 shows mean rank of arm entry over the initial 8 training trials and over the final 4 extrapolation trials. Overall mean ranks of entry into control arms are shown at each trial for comparison. Performance over the first 8 trials shows that the animals continued to track arm patterns accurately, as they had during Experiment 2. By contrast, performance on the extrapolation trials indicates that the rats did not extend tracking beyond the training trials. The extrapolation-trials curves are essentially flat. Tests of mean rank on $R$ trials versus mean rank on $N$ trials showed no significant difference for the SA pattern $(t<1.00)$ or the DA pattern $[t(3)=2.32]$. Comparisons of mean ranks on $R$ and $N$ extrapolation trials with mean rank on control arms failed to yield significant differences for any pattern (all $t \mathrm{~s}<1.00$ ).

These findings clearly indicate that rats were not able to extend patterns beyond the initial 8 trials used during acquisition. These data fail to support the arm-pattern hypothesis but are in keeping with the trial-number hypothesis, which predicts that animals should be unable to extrapolate patterns.

\section{GENERAL DISCUSSION}

These experiments provide several important findings regarding rats' pattern tracking behavior on the radial maze. First, they show us clearly that rats can track simul- taneously as many as four different patterns, even when these patterns were generated by different rules. Furthermore, the demonstration of accurate tracking was dependent upon the use of a maze in which the animals had to expend effort to climb over blocks in order to enter arms. The rats only partially tracked patterns in Experiment 1 with unimpeded access to arms, but tracking of all rewarded and nonrewarded arms appeared when blocked arms were used in Experiment 2.

As an account of the tracking observed in Experiment 2, the arm-pattern hypothesis suggests that the rats learned each of the four patterns assigned to different arms and used rules or sequential associations to predict the events on each arm. The trial-number hypothesis, on the other hand, suggests that the rats learned only the locations of the rewarded arms at each ordinal trial position. Several aspects of these findings seem to support the trial-number hypothesis more than the arm-pattern hypothesis. For one, the summation of arm vectors analysis showed that the animals became progressively more stereotyped in their sequence of responses as acquisition proceeded. Although the relationship between the development of tracking behavior and stereotypy is correlational, it seems quite possible that learning to track rewarded arms also led to increasingly stereotyped sequences of arm entrances. The further observation that the initial arm choices of a trial were most stereotyped is in keeping with the idea that the rats developed strong preferences for certain rewarded 
arms on each trial. It should also be noted that the development of a fixed pattern of arm entries is unusual behavior on the radial maze (Olton, 1979), suggesting it developed as a response to the conditions imposed on the animals by these experiments.

Pattern tracking in both Experiments 1 and 2 always consisted of the animals' dropping to ranks below controlarm level on rewarded trials but never exceeding controlarm level on nonrewarded trials. This finding gives the appearance that the difference between one and two pellets was more motivationally significant to the animals than was the difference between no pellets and one pellet. However, it may be the case that, given limited memory capacity, the rats best remembered the rewarded or two-pellet arms associated with each trial. Distinctions between further arms (containing no pellets and one pellet) might have been fuzzy, thus leading to little discrimination between them and relatively less stereotypy in order of arm entrances. The observed preference for two pellets over one pellet and the indifference between no pellets and one pellet might be a product of the unique task given the rats in these experiments and a limited memory capacity.

The final piece of evidence supporting the trial-number hypothesis is the finding that the rats failed to extrapolate patterns in Experiment 3. Although the highly trained animals showed clear tracking of SA and DA patterns over the initial 8 trials of a session, they completely failed to track these patterns further on Trials 9-12. If the animals had learned rules or sequential associations, tracking should have continued to some degree on the extended trials, particularly given the fact that rewards and nonrewards continued to be placed on arms according to the patterns. If the rats were using trial number as a cue to indicate which arms to enter first, however, they should have been at a complete loss for information on Trials $9-12$, because they had never before been trained at these trial numbers.

It could be argued in favor of the arm-pattern hypothesis that the rats came to remember arm patterns as higher order rules or integrated chunks (Capaldi, 1992), consisting of extended strings of items or even the entire 8-trial sequence. In this case, one might not expect extrapolation of the arm patterns. However, we feel that the findings of Experiment 3 combined with the stereotypy analysis and the failure of the animals to distinguish between nonrewarded and control arms favor the trial-number hypothesis.

Roberts and Dale (1981) showed that rats tested on the radial maze over repeated massed trials developed proactive interference, as shown by a drop in spatial memory accuracy after the first trial. Rats eventually overcame this drop in performance by adopting a strategy of entering adjacent alleys in a clockwise or counterclockwise direction. Dallal and Meck (1990) also found proactive interference over repeated trials but observed that a group of rats that could chunk arm visits by different food types showed strong resistance to proactive interference. In the experiments reported here, it is interesting to note that although the animals were tested over several repeated trials within a daily session, no drop across trials in memory for unentered arms was found. We suggest that the arm-number strategy used to select rewarded arms first on each trial reduced intertrial interference or proactive interference, just as the adjacent-arm strategy in the Roberts and Dale study and the chunking strategy in the Dallal and Meck experiment attenuated proactive interference effects.

The conclusion that these findings support the trialnumber hypothesis suggests that the pattern tracking seen in these experiments was more apparent than real. It appears that the rats did not learn to track four independent patterns. What the data suggest they did do was learn to keep track of the ordinal position of trials and which arms were the rewarded arms on that trial. It should be pointed out that although the trial-number hypothesis accounts for the findings of the experiments reported here, it may not account for the findings of Phelps and Roberts (1991). In the Phelps and Roberts experiments, the arm assignment of different patterns changed randomly between sessions. Phelps and Roberts found that rats selectively tracked only simple rule-driven patterns and not quasirandom patterns that were repeated on each daily session. The trial-number hypothesis suggests that rats should have learned to track all patterns of reward and nonreward, regardless of the nature of the pattern. The fact that the arm positions of patterns changed between sessions might have encouraged pattern learning in the Phelps and Roberts experiments.

Rats may adopt different solutions to different tracking problems on the radial maze. In the present experiments, a trial-number strategy might have been the easiest or most parsimonious way to learn the locations of reward on successive trials and to avoid proactive interference. In the Phelps and Roberts experiments, rats may have learned to track particular patterns. In other experiments, in which quality of food in maze arms is varied, rats learn to track food types (Dallal \& Meck, 1990). These various findings suggest that rats are capable of developing a wide variety of memory strategies or "software" suitable to the demands of the particular radial maze task they face (Rakitin, Dallal, \& Meck, 1992).

\section{REFERENCES}

Ash, M., \& RoberTs, W. A. (1992). Central-place foraging by rats on the radial maze: The effects of patch size, food distribution, and travel time. Animal Learning \& Behavior, 20, 127-134.

BloOM, J. M., \& CAPALDI, E. J. (1961). The behavior of rats in relation to complex patterns of partial reinforcement. Journal of Comparative \& Physiological Psychology, 54, 261-265.

BROWN, M. F. (1990). The effects of maze-arm length on performance in the radial-arm maze. Animal Learning \& Behavior, 18, 13-22.

Brown, M. F., \& HugGins, C. K. (1993). Maze-arm length affects a choice criterion in the radial-arm maze. Animal Leaming \& Behavior, 21, 68-72. 
Brown, M. F., \& Lesniak-Karpiak, K. B. (1993). Choice criterion effects in the radial-arm maze: Maze-arm incline and brightness. Learning \& Motivation, 24, 23-39.

CaPaldi, E. J. (1992). Levels of organized behavior in rats. In W. K. Honig \& J. G. Fetterman (Eds.), Cognitive aspects of stimulus control (pp. 385-404). Hillsdale, NJ: Erlbaum.

CAPAlDI, E. J., \& MilleR, D. J. (1988). Counting in rats: Its functional significance and the independent cognitive processes that constitute it. Journal of Experimental Psychology: Animal Behavior Processes, 14, 3-17.

CAPALDI, E. J., \& VERRY, D. R. (1981). Serial order anticipation learning in rats: Memory for multiple hedonic events and their order. Animal Learning \& Behavior, 9, 441-453.

DALE, R. H. I. (1986). Spatial and temporal response patterns on the eight-arm radial maze. Physiology \& Behavior, 36, 787-790.

Dallal, N. L., \& MECK, W. H. (1990). Hierarchical structures: Chunking by food type facilitates spatial memory. Journal of Experimental Psychology: Animal Behavior Processes, 16, 69-84.

Davis, H., \& Perusse, R. (1988). Numerical competence in animals Definitional issues, current evidence, and a new research agenda. $\mathrm{Be}$ havioral \& Brain Sciences, 11, 561-615.

Foreman, N. (1985). Algorithmic responding on the radial maze in rats does not always imply absence of spatial encoding. Quarterly Journal of Experimental Psychology, 37B, 333-358.

FounTAIN, S. B. (1990). Rule abstraction, item memory, and chunking in rat serial-pattern tracking. Journal of Experimental Psychology: Animal Behavior Processes, 16, 96-105.

Fountain, S. B., Henne, D. R., \& Hulse, S. H. (1984). Phrasing cues and hierarchical organization in serial pattern learning by rats. Journal of Experimental Psychology: Animal Behavior Processes, 10, 30-45.
Fountain, S. B., \& Hulse, S. H. (1981). Extrapolation of serial stimulus patterns by rats. Animal Learning \& Behavior, 9, 381-384.

Gallistel, C. R. (1990). The organization of learning. Cambridge. MA: MIT Press.

Higashida, A., \& OGaWA, N. (1987). Radial maze performance in three strains of rats: The role of spatial strategy. Research Communications in Psychology, Psychiatry, \& Behavior, 12, 118-128.

Olton, D. S. (1979). Mazes, maps, and memory. American Psychol ogist, 34, 583-596.

Phelps, M. T., \& Roberts, W. A. (1989). Central-place foraging by Rattus norvegicus on a radial maze. Journal of Comparative Psychology, 103, 326-338.

PhelPS, M. T., RoberTs, W. A. (1991). Pattern tracking on the radial maze: Tracking multiple patterns at different spatial locations. Journal of Experimental Psychology: Animal Behavior Processes, 17, 411-422.

Rakitin, B. C., Dallal, N. L., \& Meck, W. H. (1992). Spatial memory structure and capacity: Influences on problem-solving and memorycoding strategies. In W. K. Honig \& J. G. Fetterman (Eds.), Cognitive aspects of stimulus control (pp. 155-183). Hillsdale, NJ: Erlbaum.

ROBERTS, W. A., \& DALE, R. H. I. (1981). Remembrance of places lasts: Proactive inhibition and patterns of choice in rat spatial memory. Learning \& Motivation, 12, 261-281.

ROBERTS, W. A., \& ILERSICH, T. J. (1989). Foraging on the radial maze: The role of travel time, food accessibility, and the predictability of food location. Journal of Experimental Psychology: Animal Behavior Processes, 15, 274-285.

(Manuscript received March 4, 1993; revision accepted for publication September 14, 1993.) 